\title{
Paciente en diálisis con lesiones por calcifilaxis
}

\author{
Jessica Ferre Vargas, Francisco Manuel Fernández Rienda, Mirco Dal Maso, Carmen Purificación Rodríguez \\ García, Ángela Pérez González, Delia Pulido Villa
}

\section{Centro de Diálisis Nuestra Señora de la Cabeza. Diaverum. Motril. Granada}

\section{Intgroducción:}

La calcifilaxis se caracteriza por isquemia y necrosis cutánea secundaria a la calcificación, fibrosis y trombosis de pequeñas arteriolas dermoepidérmicas. Esta enfermedad afecta entre el 1 y el $4 \%$ de los pacientes en diálisis. El estudio se centra en el caso clínico de una paciente de 44 años de edad en tratamiento sustitutivo desde 1999. En diciembre 2011 inicia dolor en ambos M MII con presencia de nódulos dolorosos, apareciendo posteriormente úlceras necróticas por calcifilaxis. Se presenta la evolución de la paciente según el tratamiento administrado, los productos utilizados para la cura de las heridas, la medicación intravenosa y la pauta de hemodiálisis. Se utilizan gráficos donde se representan niveles analíticos, lesiones, intervenciones quirúrgicas tratamiento intravenoso y evolución del nivel del dolor. Se documenta con fotos la evolución de las lesiones. A pesar del mal pronóstico de la enfermedad en este caso se ha conseguido la curación completa de las lesiones en un periodo de 11 meses con una significativa mejora de la calidad de vida de la paciente.

\section{Referencias Bibliográficas}

1. Daniel Lerma García, Beatriz Lerma García, Miren Iosune Echeverría, Eciolaza Joan Sureda Parera, Hospital Can Misses. Ibiza. Cuidados integrales de enfermería a pacientes con úlceras por calcifilaxis en tratamiento con Hemodiálisis: a propósito de un caso. Obtenido en: http://www.revistaseden.org/ files/2124_P\%C3\%Alginas\%20de\%202009-9. pdf, (online) Ultima Consulta: 20/03/2012.

2. L.Salanova Villanueva, M. C. Sánchez González, J.A. Sánchez Romero, P. Sanz. Tratamiento exitoso con tiosulfato sódico en la arteriolopatía urémica calcificante. Nefrología (Madr.) vol.31 no.3 Madrid 2011, Cartas al director.

3. 0. Conde Rivera, M. Camba Caride, E. Novoa Fernández, M. Borrajo Prol, J. Santos Nores, E. Iglesias Lamas, W. López Alarcón. Servicio de Nefrología. Complejo Hospitalario de Ourense. Tratamiento multidisciplinar: Una opción terapéutica en la calcifilaxis Nefrologia 2011;31(5):614-6 doi: 10.3265/Nefrologia.pre2011.Jun.10954.

4. Rodríguez-Villarreal, R. Callejas, M. Sánchez, N. Laso, P. Gallar, 0. Ortega, M. Ortiz, C. Mon, J.C. Herrero, A. Oliet, C. Di Giogia, A. Vigil. Calcifilaxis 0 arteriolopatía urémica calcificante: combinación de tratamientos NefroPlus 2010;3(3):39-44 | Doi. 10.3265/NefroPlus.pre2010.Jul.10502.

5. José V. Torregrosa, Carlos E. Durán, Xoana Barros, Miquel Blasco, Marta Arias, Aleix Cases, Josep M. Campistol, Servicio de Nefrología Hospital Clinic Barcelona. Tratamiento eficaz de la arteriolopatía urémica calcificante con bifosfonatos Nefrología 2012; 32(3):329-334 | Doi. 10.3265/Nefrología. pre2012.Jan.11137.

6. José-Vicente Torregrosa, Jordi Bover, Jorge Cannata Andía, Víctor Lorenzo, ALM de Francisco, Isabel Martínez, Mariano Rodríguez Portillo, Lola Arenas, Emilio González Parra, Francisco Caravaca, Alejandro Martín-Malo, Elvira Fernández Giráldez, Armando Torres.

7. Recomendaciones de la Sociedad Española de Nefrología para el manejo de las alteraciones del metabolism óseo-mineral en los pacientes con enfermedad renal crónica (S.E.N.-MM) Nefrología 2011;31(Suppl.1):3-32 | doi:10.3265/Nefrología. pre2011.Jan.10816. 